\title{
ALTERAÇÕES DE USO E COBERTURA DA TERRA NA BACIA HIDROGRÁFICA DO RIO GUALAXO DO NORTE - MG: 2008 A 2018
}

\author{
Dayane Caroline Freitas Carvalho \\ Universidade Federal de Minas Gerais, Instituto de Geociências, Belo Horizonte, MG, Brasil \\ dayanecfc@gmail.com \\ Adriana Monteiro da Costa \\ Universidade Federal de Minas Gerais, Instituto de Geociências, Belo Horizonte, MG, Brasil \\ drimonteiroc@yahoo.com.br \\ João Herbert Moreira Viana \\ Embrapa Milho e Sorgo, Sete Lagoas, MG, Brasil \\ joao.herbert@embrapa.br
}

\begin{abstract}
RESUMO
O mapeamento das alterações das classes de uso e de cobertura da terra é um instrumento primordial para a análise do território. Este estudo analisou as alterações de uso e cobertura da terra na bacia hidrográfica do rio Gualaxo do Norte - MG com o propósito de identificar e avaliar as principais classes de uso e cobertura nos últimos dez anos (2008 a 2018), destacando as alterações causadas pelo rompimento da barragem de Fundão e os padrões de organização de uso e de cobertura da terra existentes na bacia. Foram realizadas as classificações de imagens de satélite referentes aos anos de 2008, 2015 e 2018 pelo método de classificação supervisionada orientada a objeto com o algoritmo do Nearest Neighbor (vizinho mais próximo), considerando, forma, tamanho e compacidade. Assim, foi verificado que há oito classes de uso e de cobertura da terra principais, que no intervalo de dez anos, sofreram pequenas ou grandes mudanças. Também se constatou que a bacia retrata três grandes ambientes, o primeiro marcado pela atividade minerária, localizado no alto curso, o segundo pela silvicultura e formação florestal, no médio curso, e o terceiro pelas áreas campestres, incluindo diversas áreas de pastagem no baixo curso.
\end{abstract}

Palavras-chave: Sensoriamento remoto. Barragem de Fundão. Classificação orientada a objeto.

\section{CHANGES OF USE AND COVERAGE OF THE SOIL IN RIVER BASIN GUALAXO DO NORTE - MG: 2008 TO 2018}

\begin{abstract}
Mapping the change of the land use and of the land cover is an essential tool for territory analysis. This paper analyzed the changes in the land use and in the land cover in the Gualaxo do Norte river basin in Minas Gerais (Brazil), aiming to identify and to analyze the main land use and land cover classes in the past decade (2008-2018) and highlighting the changes brought on by the Fundão mining dam rupture, as well as the land use and land cover organization patterns in the basin. Satellite image classification was performed for the years 2008, 2015 and 2018, through object-oriented supervised classification using the Nearest Neighbor algorithm, with the parameters such as shape, size and compacity. Thereby eight main land use and land cover classes were found in the 10 years analyzed, which have gone through major or minor changes. We have also found that the river basin displays three great environments, the first predominantly represented by the mining activities, in the upper course; the second represented by the silviculture and the forest formation in the middle course and the third typically made of represented by grassland in many areas of pastures in the lower course.
\end{abstract}

Keywords: Remote sensing. Fundão dam. Object-oriented classification. 


\section{INTRODUÇÃO}

As transformações antrópicas na paisagem podem comprometer a qualidade e disponibilidade de recursos essenciais à vida, como o solo, a cobertura vegetal e os corpos d'água (CARMO, 2009). Deste modo, estudar as dinâmicas e as alterações do uso e da cobertura da terra contribui para o entendimento de questões dos mais diversos interesses que podem ser desde o caráter histórico, ambiental, econômico, e estrutural até o político de decisão. Assim, avaliar quais mudanças e quais aspectos atuaram e atuam sobre as paisagens é de fundamental importância no subsídio às tomadas de decisão na gestão dos territórios.

O mapeamento e a análise das alterações das classes de uso e de cobertura da terra é um instrumento primordial para a compreensão dos padrões de organização do espaço, uma vez que suas tendências possam ser analisadas. Este monitoramento consiste em buscar conhecimento de toda a sua utilização por parte do homem ou, quando não utilizado pelo homem, a caracterização de tipos de categorias de vegetação natural que revestem o solo, como também de suas respectivas localizações. De forma sintética, a expressão "uso da terra ou uso do solo" pode ser entendida como a forma pela qual o espaço está sendo ocupado pelo homem (ROSA, 2007).

A partir da década de 1970, foram registrados grandes avanços nas técnicas para análise de classificação de uso e de cobertura da terra. Atualmente, existem diversos métodos e algoritmos de classificação, com inúmeras especificidades e desempenhos (PHIRI e MORGENROTH, 2017). Dentre estes, pode-se citar o método da classificação orientada a objetos (OBIA) que utiliza objetos geográficos como unidades básicas para classificação da cobertura da terra (PEÑA et al., 2014; DORREN; MAIER; SEIJMONSBERGEN, 2003). Essa abordagem reduz a variação dentro da classe e geralmente remove os efeitos que resultam de pixels isolados, principalmente devido à classificação errônea. A OBIA tem uma grande vantagem, pois incorpora diversas fontes de informação como textura, forma e posição como base para a classificação (LI et al., 2014; HUSSAIN et al., 2013; MOSKAL; STYERS; HALABISKY, 2011).

Geralmente, a OBIA é aplicada em imagens de alta resolução. Entretanto, existem estudos com a aplicação do método em imagens de média resolução, como Landsat (SAMAL e GEDAM, 2015). Em estudos realizados para comparar o desempenho da classificação orientada a objetos com a pixel a pixel de imagens Landsat, verificou-se que a OBIA produziu precisões de classificação mais altas em vários tipos de cobertura da terra (GILBERTSON; KEMP; VAN NIEKERK, 2017; CAI e LIU, 2013; FLANDERS; HALL-BEYER; PEREVERZOFF, 2003).

Os impactos gerados pelas interferências antrópicas nas paisagens têm sido cada vez mais frequentes. Dentre estes, destacam-se os da construção das barragens de rejeitos da mineração, que interferem substancialmente nos processos fluviais, na erosão, no transporte e na deposição de sedimentos ao longo do canal principal e junto à desembocadura. Tais alterações, podem, a médio e a longo prazo, desencadear ou intensificar efeitos adversos, como o aumento dos processos erosivos nas margens e o assoreamento dos canais fluviais, as alterações na flora e na ictofauna, além de comprometer hábitos de comunidades dependentes do rio, como, por exemplo, ribeirinhos e pescadores (COELHO, 2007).

Nos últimos três anos, o impacto destas estruturas afetou o estado de Minas Gerais, e registrou dois grandes desastres provocados pelo rompimento de barragens de rejeitos (Barragem de Fundão em Mariana, em 2015 e, Barragem do Córrego do Feijão em Brumadinho, em 2019). O processo alterou toda uma paisagem natural e social das áreas diretamente afetadas, resultando em grandes volumes de rejeitos descarregados nos canais fluviais, o que está comprometendo a dinâmica de inúmeras bacias hidrográficas das regiões.

O rompimento da barragem de rejeitos de Fundão no município de Mariana em Minas Gerais é considerado o mais grave episódio dessa natureza no mundo (LACAZ; PORTO; PINHEIRO, 2017). Com um volume total de cerca de 50 milhões de metros cúbicos de rejeito de mineração, foram liberados da barragem 34 milhões de metros cúbicos, que galgaram uma segunda barragem a jusante, a de Santarém, sendo o rejeito lançado na bacia hidrográfica do rio Doce. A avalanche de lama deixou um rastro de destruição, marcado por perdas de vidas humanas, severa alteração biofísica, e incalculável perda patrimonial no sentido amplo. A corrente fluvial com altíssima turbidez percorreu o restante do fundo de vale por, aproximadamente, $115 \mathrm{~km}$ até alcançar a represa da Usina Hidrelétrica de Candonga / Risoleta Neves (barragem de Candonga), "empurrando" uma onda de cheia, que seguiu destruindo, o que encontrava no canal e sobre os baixos terraços e encostas, que constituem as margens dos rios Gualaxo do Norte e do Carmo (SAADI e CAMPOS, 2015). 
Os impactos gerados pelo rompimento refletiram diretamente não só na queda da qualidade dos recursos hídricos, como também na sedimentação do material sobre as margens dos rios, soterrando o leito menor e, em alguns locais, até além dele, promovendo intensificação dos processos erosivos, com remoção de vegetação ciliar (VIANA e COSTA, 2016).

Diante deste contexto, o objetivo deste trabalho foi identificar e analisar as mudanças no uso e na cobertura da terra dos últimos dez anos (2008 - 2018), na bacia hidrográfica do rio Gualaxo do Norte - MG destacando os padrões de organização de uso e cobertura da terra existentes na bacia, com foco nas áreas afetadas pelo desastre.

\section{MATERIAIS E MÉTODOS}

\section{Localização e caracterização da área}

A Bacia Hidrográfica do rio Gualaxo do Norte está localizada na região central do estado de Minas Gerais. Em sua extensão, a bacia engloba parte dos municípios de Ouro Preto (78 km²), Mariana (375 $\mathrm{km}^{2}$ ) e Barra Longa $\left(107 \mathrm{~km}^{2}\right)$, somando uma área de aproximadamente $560 \mathrm{~km}^{2}$ (IBGE, 2006) (Figura 1). O rio Gualaxo do Norte tem aproximadamente $87 \mathrm{~km}$ de extensão, e deságua no rio do Carmo, afluente do rio Doce, pertencendo à Unidade Planejamento de Recursos Hídricos DO1 - Rio Piranga (IGAM, 2010).

Figura 1 - Mapa de localização da bacia hidrográfica do rio Gualaxo do Norte.

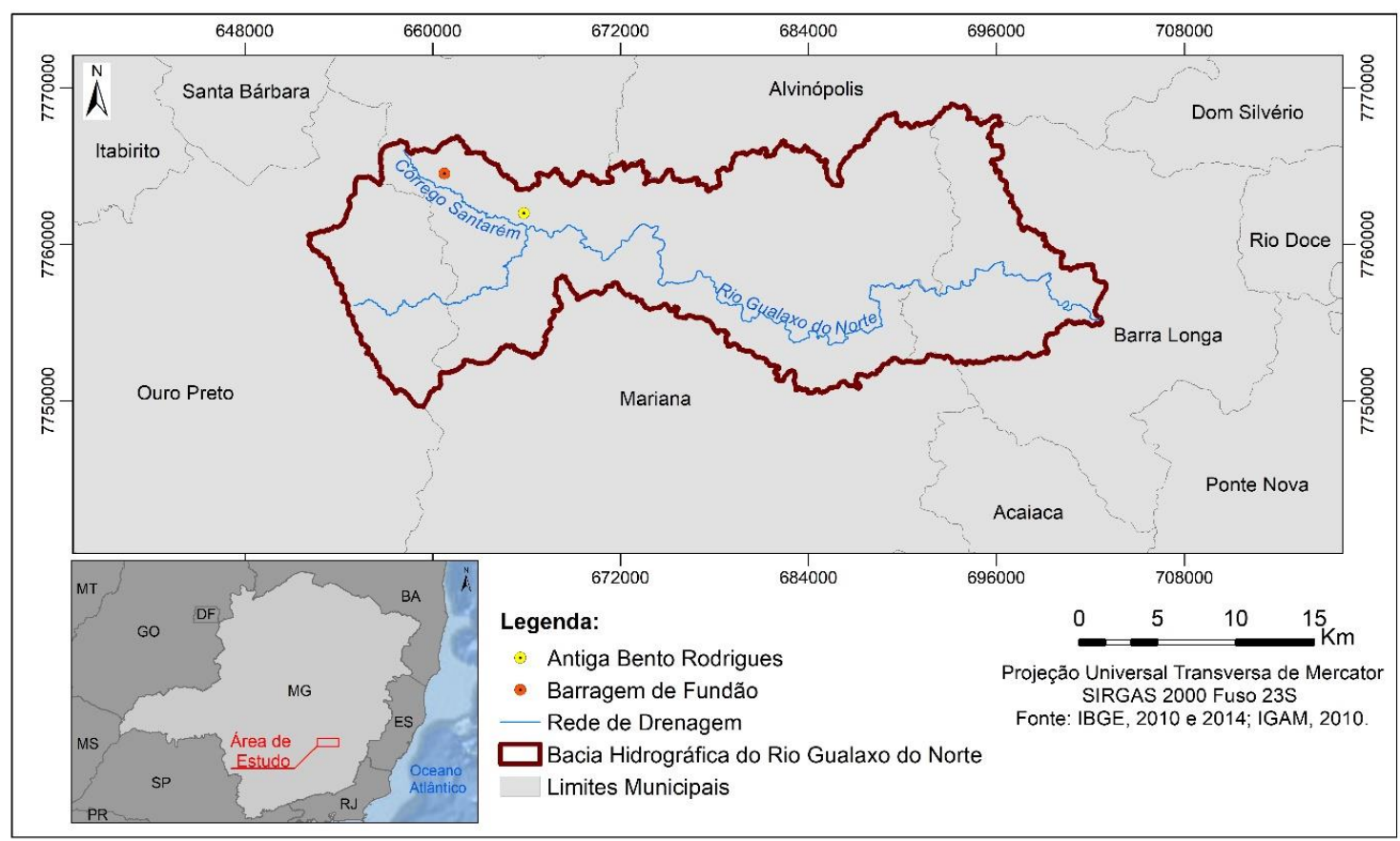

$\mathrm{Na}$ bacia, a montante, predominam os afloramentos rochosos e as classes de solos dos Cambissolos Háplicos Tb Distrófico e Distroférrico e, no baixo curso, a classe dos Argissolos Vermelhos Eutróficos. Entretanto, a classe mais representativa da bacia são os Latossolos Vermelho-Amarelo Distrófico (EMBRAPA, 1979).

A Litologia da bacia é composta por itabiritos, dolomitos e filitos em seu alto curso, área com diversas minas de minério de ferro e suas respectivas barragens de rejeitos. A barragem de Fundão se insere neste contexto, estando localizada mais precisamente no córrego Santarém, afluente do rio Gualaxo do Norte (SANTOS, et al. 2017). No médio curso, aparecem rochas do Supergrupo Rio das Velhas, como quartzitos e xistos, e do Complexo Santa Bárbara, como gnaisses migmatíticos e granitos. O baixo curso é composto por rochas do Complexo Acaiaca, como, gnaisses, quartzitos e migmatitos e do Complexo Mantiqueira, com gnaisses e pegmatitos (CONSÓRCIO ECOPLAN-LUME, 2010). 
A geomorfologia da bacia conta com quatro unidades morfopedológicas segundo Saadi e Campos (2015), sendo elas: as escarpas do Caraça, apresentando escarpas rochosas elevadas e subverticais; os morros de Mariana, com morros e colinas com altura inferior a 200 metros (Figura 2), cristas esparsas e vales encaixados; as colinas do alto Piracicaba, com colinas convexas com altura inferior a 200 metros, cristas esparsas e vales encaixados; e o baixo rio do Carmo, com morros convexos com altura superior a 200 metros e vales muito encaixados.

Figura 2 - Morros e colinas na bacia hidrográfica do rio Gualaxo do Norte.

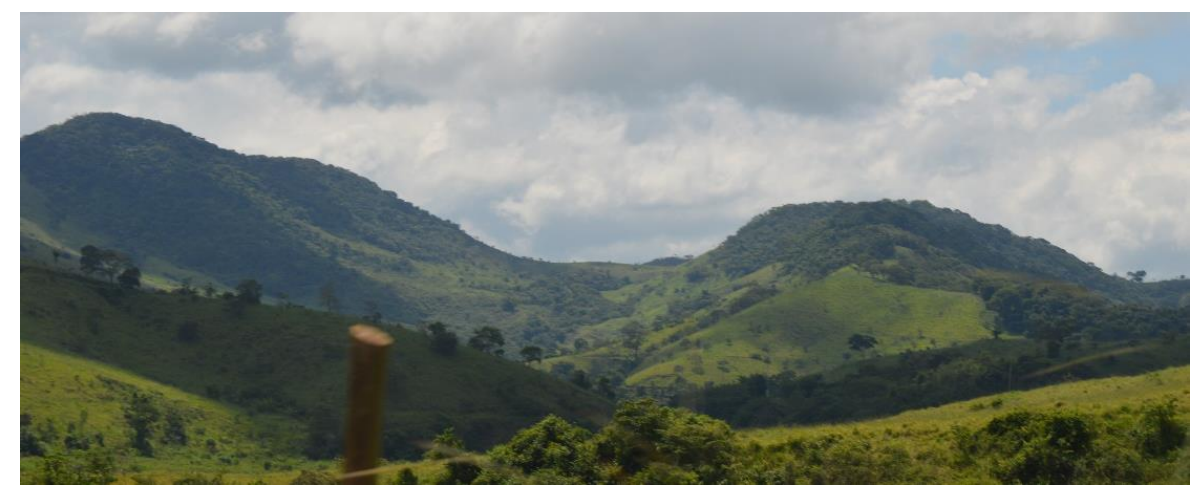

Fonte - Adriana Monteiro.

\section{Materiais e bases de dados}

Os materiais utilizados nesse estudo, foram:

a) Software ArcGIS versão 10. 3 (ESRI, 2014);

b) Software QGIS - versão 2.18.13 (QGIS DEVELOPMENT TEAM, 2016);

c) Software Google Earth Pro ${ }^{\circledR}$ 7.1.8.3036 (32-bit), 2017;

d) Software Ecognition Developer Trial 8.0. (TRIMBLE 1995-2011);

e) Imagens do Satélite Sentinel-2, obtidas gratuitamente no site do Earth Explorer (ESA, 2018);

f) Imagens do Satélite Landsat-5, obtidas gratuitamente no site do GloVis - Global Visualization Viewer (USGS, 2018);

g) Imagens do Satélite Landsat-8, obtidas gratuitamente no site do GloVis - Global Visualization Viewer (USGS, 2018);

h) Modelo Digital de Elevação ALOS PALSAR com resolução espacial de 12,5 metros (JAXA/METI, 2010);

i) GPS Garmin Map 64s e

j) Câmera Fotográfica.

Os materiais primários utilizados foram imagens de satélite com resolução espacial de 30 (Landsat-5 e Landsat-8) e 10 (Sentinel-2) metros da área de estudo numa perspectiva temporal de 10 anos, referentes aos anos de 2008, 2015 e 2018.

Os materiais utilizados referem-se às imagens gratuitas e disponíveis na plataforma Earth Explorer e Glovis, geridas pelo Serviço Geológico dos Estados Unidos (USGS). Os equipamentos e softwares utilizados foram disponibilizados pelo Laboratório de Solos e Meio Ambiente do Instituto de Geociências da Universidade Federal de Minas Gerais.

Após a aquisição dos materiais, foram realizados o processamento dos dados, as visitas de reconhecimento e de checagem em campo e, a geração dos resultados e da discussão.

Foram utilizadas imagens de satélites diferentes devido o intervalo de tempo estudado (2008 a 2018). A seleção das datas das cenas ocorreu partir de três critérios: a) os acontecimentos chave; b) a 
existência de imagens nos demais anos, com datas próximas, e, c) a presença de pouca ou nenhuma nuvem. Os acontecimentos chave foram: a existência de área rural consolidada, definida no artigo 3으, inciso III, Lei no 12.651/12 (Código Florestal) (BRASIL, 2012), como área de imóvel rural com ocupação antrópica preexistente a 22 de julho de 2008; o rompimento da barragem de Fundão, em 05 de novembro de 2015, e; o cenário atual, no ano de 2018.

A imagem do satélite Landsat- 5 foi utilizada para o mapeamento referente ao ano de 2008, com data de aquisição da imagem de 20 de agosto de 2008. A escolha ocorreu devido à baixa qualidade das imagens de julho, mês em que a Lei № 12.651/12 entrou em vigor. Foram utilizadas as bandas 1, 2, 3, (bandas do RGB -vermelho, verde e azul) e 4 (NIR -infravermelho próximo). A Tabela 1 apresenta as principais características das bandas utilizadas.

Tabela 1 - Bandas utilizadas na classificação da imagem Landsat-5 e suas principais características.

\begin{tabular}{cccc}
\hline Banda & $\begin{array}{c}\text { Intervalo Espectral } \\
(\boldsymbol{\mu m})\end{array}$ & $\begin{array}{c}\text { Região do } \\
\text { Espectro }\end{array}$ & $\begin{array}{c}\text { Resolução } \\
\text { Espacial }(\mathbf{m})\end{array}$ \\
\hline $\mathbf{1}$ & $0,45-0,52$ & Azul & $\mathbf{3 0}$ \\
$\mathbf{2}$ & $0,52-0,60$ & Verde & $\mathbf{3 0}$ \\
$\mathbf{3}$ & $0,63-0,69$ & Vermelho & $\mathbf{3 0}$ \\
$\mathbf{4}$ & $0,76-0,90$ & NIR & $\mathbf{3 0}$
\end{tabular}

Fonte - Adaptado do Departamento de Geração de Imagens (INPE, 2018).

A imagem do satélite Landsat-8 foi utilizada para o mapeamento referente ao ano de 2015 , com data de aquisição da imagem de 25 de setembro de 2015. Foram utilizadas as bandas 2, 3, 4 (bandas do RGB -vermelho, verde e azul) e 5 (NIR -infravermelho próximo). A Tabela 2 apresenta as principais características das bandas utilizadas.

Tabela 2 - Bandas utilizadas na classificação da imagem Landsat-8 e suas principais características.

\begin{tabular}{cccc}
\hline Banda & Intervalo Espectral $(\boldsymbol{\mu m})$ & Região do Espectro & $\begin{array}{c}\text { Resolução Espacial } \\
(\mathbf{m})\end{array}$ \\
\hline $\mathbf{2}$ & $0,45-0,51$ & Azul & $\mathbf{3 0}$ \\
$\mathbf{3}$ & $0,52-0,59$ & Verde & $\mathbf{3 0}$ \\
$\mathbf{4}$ & $0,64-0,67$ & Vermelho & $\mathbf{3 0}$ \\
$\mathbf{5}$ & $0,85-0,88$ & NIR & $\mathbf{3 0}$ \\
\hline
\end{tabular}

Fonte - USGS (2018).

A imagem do satélite Sentinel-2 foi utilizada para o mapeamento referente ao ano de 2018, com data de aquisição da imagem de 01 de setembro de 2018. Foram utilizadas as bandas 2, 3, 4 (bandas do RGB - vermelho, verde e azul) e 8 (NIR - infravermelho próximo). A Tabela 3 apresenta as principais características das bandas utilizadas.

Tabela 3 - Bandas utilizadas na classificação da imagem Sentinel-2 e suas principais características.

\begin{tabular}{cccc}
\hline Banda & $\begin{array}{c}\text { Comprimento de onda } \\
\text { central }(\mathbf{n m})\end{array}$ & Região do Espectro & $\begin{array}{c}\text { Resolução Espacial } \\
(\mathbf{m})\end{array}$ \\
\hline $\mathbf{2}$ & 490 & Azul & 10 \\
$\mathbf{3}$ & 560 & Verde & 10 \\
$\mathbf{4}$ & 665 & Vermelho & 10 \\
$\mathbf{8}$ & 842 & NIR & 10 \\
\hline
\end{tabular}

Fonte - ESA (2018).

$\begin{array}{lllll}\text { Caminhos de Geografia } & \text { Uberlândia-MG } & \text { v. } 21, \text { n. } 76 & \text { Ago/2020 } & \text { p. } 57-71\end{array}$




\section{Definição das classes de uso e de cobertura da terra}

As classes de uso e de cobertura da terra foram definidas a partir da análise visual das imagens Landsat5, Landsat-8 e Sentinel-2, consulta ao acervo de imagens do Google Earth Pro e de visitas de campo, onde aconteceu o reconhecimento da área de estudo e a identificação dos alvos presentes nas cenas.

As classes definidas foram: afloramento rochoso, área urbana, corpos d'água, formação florestal, formação campestre, mineração, e solo exposto (Tabela 4).

Tabela 4 - Classes de uso e cobertura da terra.

\begin{tabular}{lll}
\hline Classe & Exemplo & Descrição \\
\hline
\end{tabular}

Afloramento

Área urbana

Corpos d'água

Formação florestal

Formação campestre

Mineração

Solo exposto
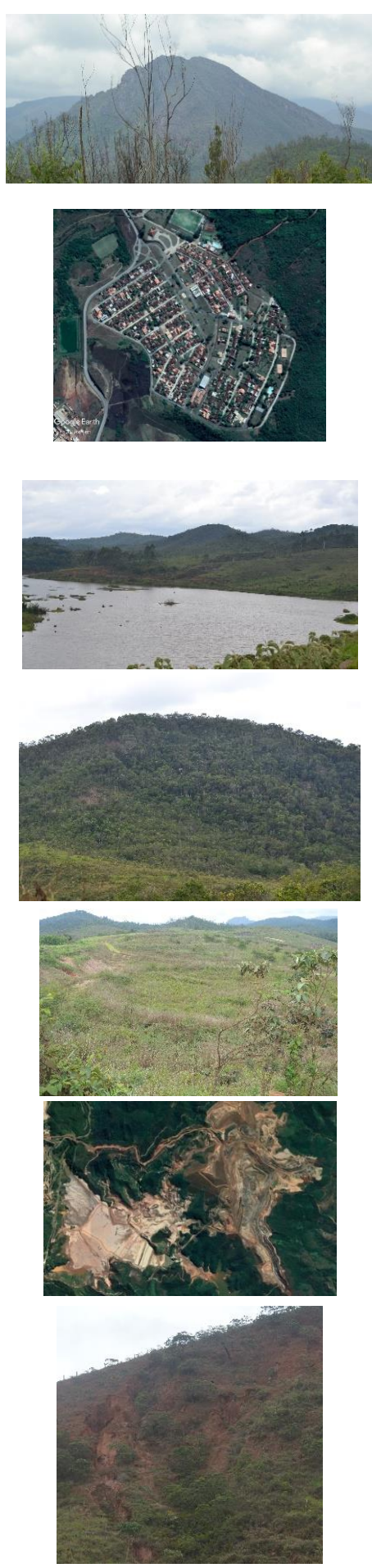

\section{Exposição rochosa na superfície.}

Área caracterizada por pavimentação e edificações contínuas e demais construções.

Caracterizados por lagos, lagoas, rios e reservatórios artificiais.

Vegetação composta por matas secundárias em estágio inicial e médio de regeneração.

Vegetação herbáceo-arbustiva compostas por árvores espaçadas e predomínio do estrato herbáceo

, incluindo as pastagens.

Processos e atividades referentes a mineração, predominando as cavas de minas.

Solo desnudo e/ou sem cobertura. 


\section{Visitas de campo e coletas de dados}

Foram realizadas duas visitas a campo, em março e em novembro de 2018, onde foram caracterizados os alvos de interesse e coletados pontos de controle, através de GPS e de registro fotográfico (Figura 3). Os pontos de controle foram utilizados como validação do mapeamento.

Figura 3 - Exemplos de diferentes ambientes na bacia.
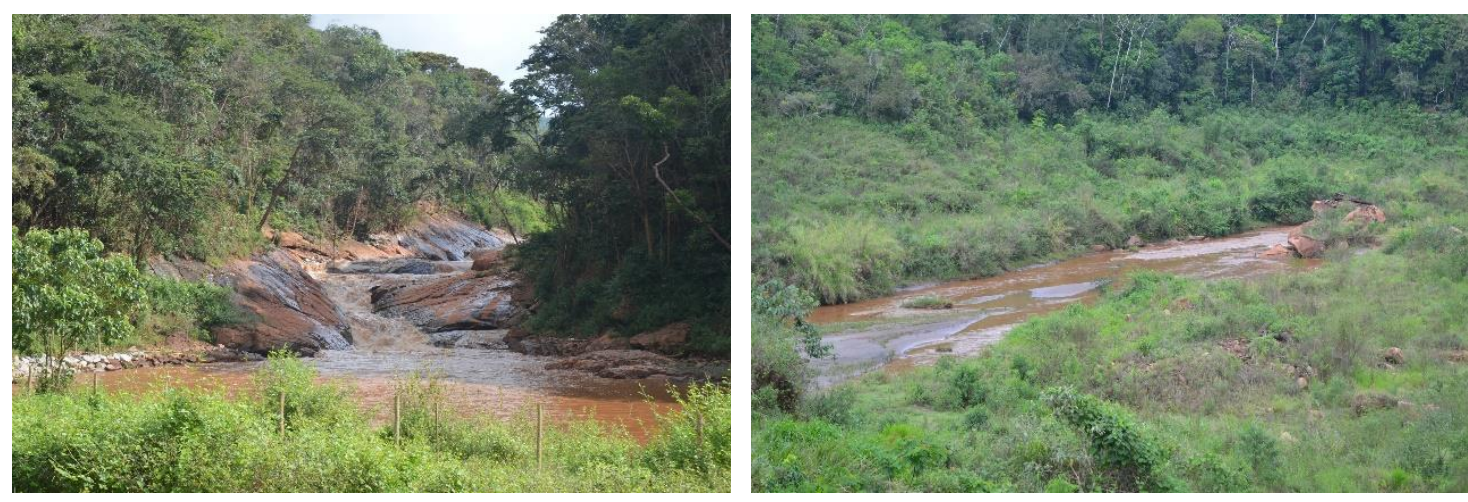

Fonte - Adriana Monteiro.

A área foi percorrida buscando-se a representação dos diferentes ambientes presentes na bacia, sendo que a dificuldade de acesso e/ou restrição não permitiu a amostragem aleatória (Figura 4).

Figura 4 - Espacialização dos pontos de controle.

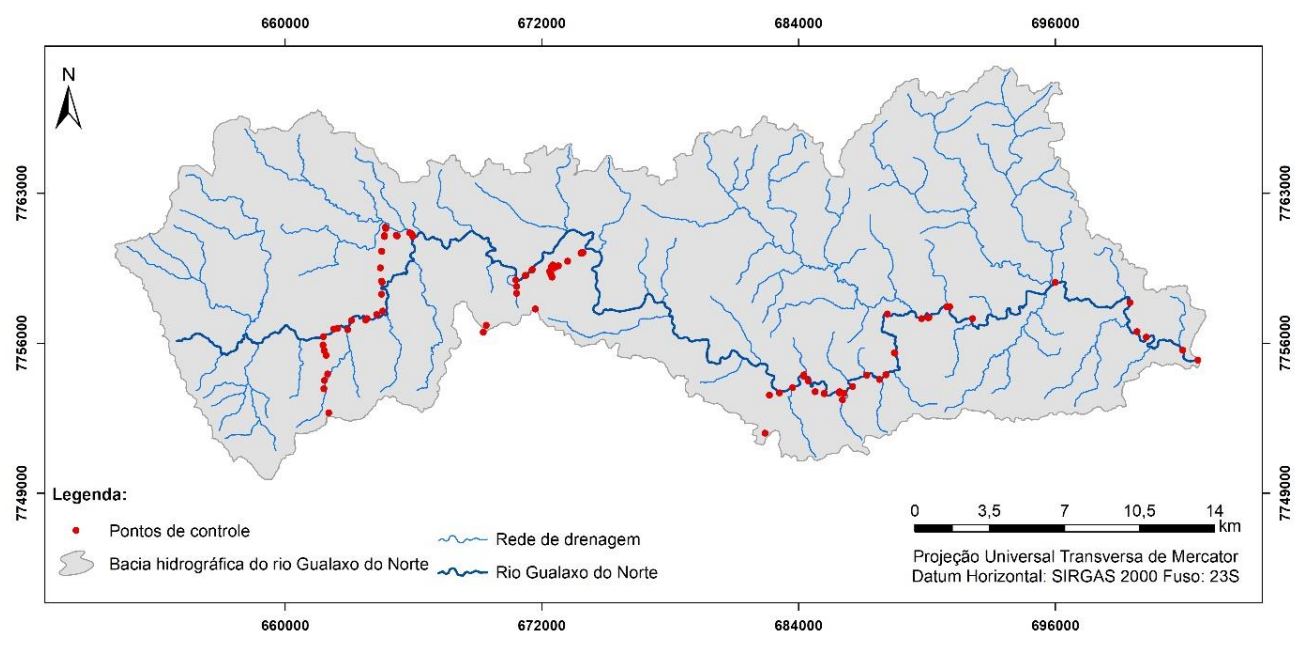

\section{Processamento das imagens}

Para o processamento das imagens foram utilizados os seguintes softwares: ArcGIS, Ecognition, QGIS e Google Earth Pro. Inicialmente, as imagens foram reprojetadas para o datum SIRGAS 2000 23S. Em seguida, o DN (digital number), indicador de nível de cinza que varia do preto ao branco de cada imagem, foi transformado em refletância. Esse processo contribui para que o efeito da variação de brilho das cenas, em alvos de natureza similar, seja minimizado, propiciando maior qualidade ao processo de classificação da imagem.

Com as imagens transformadas em refletância, iniciou-se o processo de segmentação da imagem. O processo de segmentação é um passo crucial na OBIA.

Deste modo, foi criada uma árvore de processos para a aplicação do algoritmo de segmentação multiresolução (Multiresolution Segmentation). Este algoritmo, utiliza a técnica de fusão de regiões 
emparelhadas (pairwise), no qual pares ou regiões de pixels são fundidos em unidades maiores através de interações por meio do algoritmo Fractal Net Evolution Approach (FNEA), desde que o limiar superior de homogeneidade não seja excedido (DEFINIENS, 2009; BAATZ e SCHAPE, 2000).

A segmentação multiresolução foi estabelecida a partir da combinação de três parâmetros: forma, compacidade e escala dos objetos. No campo "forma" foram definidos valores entre 0 e 1 (mínimo e máximo disponível), que modificam a relação entre os critérios de homogeneidade espacial (1) e informação espectral (0). O mesmo intervalo foi utilizado para o parâmetro "compacidade", que modifica critérios de contorno (variação entre suave (0) e brusco (1)) (PIAZZA e VIBRANS, 2014). O parâmetro "escala" define o tamanho médio dos objetos, gerados pela fusão dos pixels.

A alteração dos valores de cada parâmetro, assim como a sinergia entre eles, gerou diferentes cenários de segmentação. Entretanto, como o objetivo era evidenciar os diferentes alvos independentemente da classe, optou-se por adotar valores baixos para forma e compacidade, fornecendo assim prioridade à suavidade de contornos e da cor (informação espectral) dos objetos. Os valores adotados estão representados na Tabela 5.

Tabela 5 - Valores dos atributos do algoritmo Multiresolution Segmentation.

\begin{tabular}{ccc}
\hline Forma & Compacidade & Escala \\
\hline $\mathbf{0 , 3}$ & 0,5 & 30 \\
\hline
\end{tabular}

Em seguida, aplicou-se o segundo algoritmo de segmentação, denominado "diferença espectral" (Spectral Difference). Este algoritmo funde objetos vizinhos criados na etapa anterior, de acordo com uma média espectral, abaixo do limite dado (diferença espectral máxima), sendo mesclados para produzir os objetos finais. A diferença espectral utilizada neste trabalho foi de 0,02 , por se tratar de dados de refletância. A Figura 5 apresenta em detalhe a aplicação do algoritmo de diferença espectral.

Figura 5 - Imagem 1: Segmentação por multiresolução; Imagem 2: Segmentação por diferença espectral.
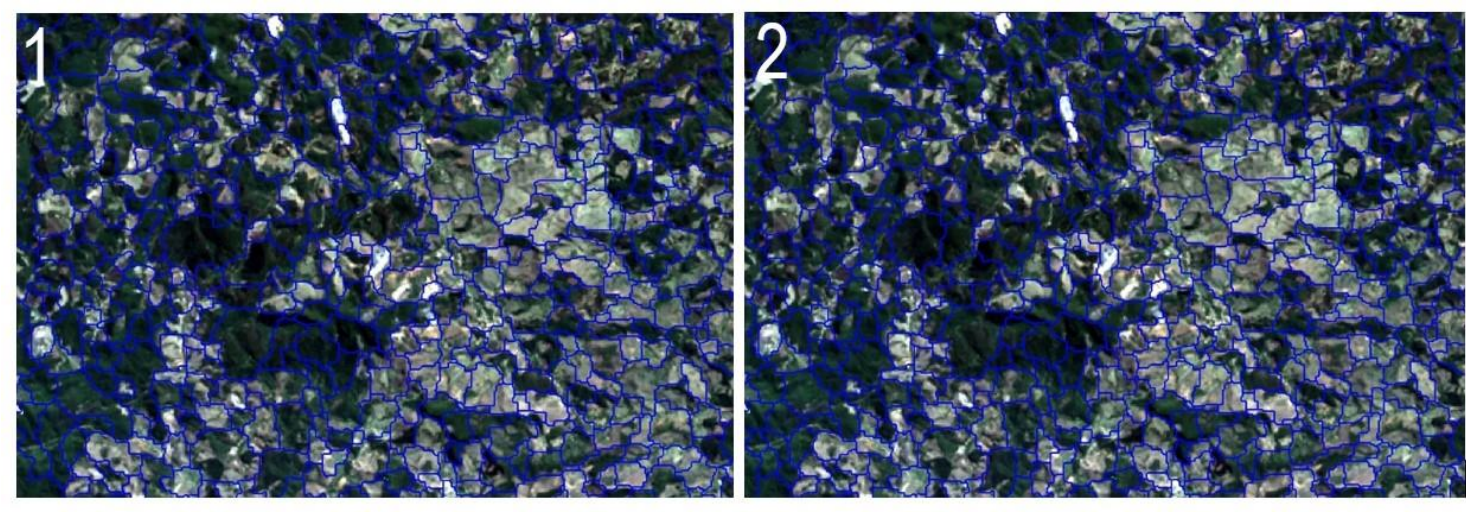

\section{Classificação orientada a objetos}

Após a segmentação das imagens, iniciou-se a etapa de coleta de amostras de teste e treinamento. As amostras de teste foram coletadas para cada classe, servindo de padrão para o algoritmo de classificação que foi aplicado posteriormente. As amostras de treinamento também foram coletadas para cada uma das classes, para a criação de uma máscara de área de teste e treinamento (TTA mask), usada posteriormente para validação e geração do índice Kappa (CONGALTON e GREEN, 1999).

Para realizar a classificação supervisionada, foi utilizado o algoritmo Nearest Neighbor - NN (vizinho mais próximo). O classificador se baseou nas amostras de teste coletadas anteriormente. Assim, o objeto mais próximo foi atribuído a uma determinada classe. 


\section{Validação}

Para avaliar a acurácia dos mapas temáticos, foram utilizadas as amostras da TTA mask. Em sequência, foi construída a matriz de erros por meio das estatísticas de estabilidade da classificação. $\mathrm{Na}$ matriz, foram avaliados os erros de omissão (amostras que não foram classificadas de acordo com as classes de referência), os erros de comissão (amostras de referência classificadas erroneamente como pertencentes a outras classes), exatidão global e índice Kappa.

Para se tornar uma classificação válida, a classificação deveria apresentar um valor de índice Kappa (Tabela 6) maior que 0,60.

Tabela 6 - Intervalos do índice Kappa.

\begin{tabular}{cc}
\hline Intervalo Kappa & Interpretação \\
$<0,00$ & Ausência de concordância \\
$0,00-0,20$ & Concordância pobre \\
$0,21-0,40$ & Concordância leve \\
$0,41-0,60$ & Concordância moderada \\
$0,61-0,80$ & Concordância substantiva \\
$0,81-1,00$ & Concordância quase perfeita \\
\hline
\end{tabular}

Fonte - Landis e Koch (1977).

As classificações válidas foram revisadas com auxílio do Google Earth Pro. Os objetos identificados como erros de omissão foram corrigidos manualmente no software ArcGIS. Por fim, foram elaborados os layouts, os cálculos de áreas e a discussão dos resultados.

\section{RESULTADOS E DISCUSSÕES}

O mapeamento do uso e de cobertura da terra da bacia hidrográfica do rio Gualaxo do Norte - MG permitiu classificar a área em oito classes distintas pré-definidas, sendo elas: afloramento rochoso, área urbana, corpos d'água, formação campestre, formação florestal, mineração, silvicultura e solo exposto.

Os dados classificados referentes ao ano de 2008 apresentaram valores de $94 \%$ para a precisão geral e 0,89 para o índice Kappa.

A matriz de confusão demostrou que não houve erros de omissão na classificação, ou seja, todas as amostras foram classificadas de acordo com as classes de referência. Em contra partida, existiram erros de comissão. Os maiores erros de comissão aconteceram nas classes dos corpos d'água e de solo exposto. A classe de corpos d'água não foi identificada pelo classificador. Deste modo, posteriormente foi vetorizada e incluída de forma manual na classificação final. A classe de solo exposto foi bastante confundida com a classe de mineração. Sendo assim, a mesma obteve isoladamente um índice Kappa de 0,53, considerada uma concordância moderada. A confusão entre as classes pode ser explicada pela refletância correlata.

A classificação da imagem de setembro de 2015 apresentou valores de precisão geral de $99 \%$ e índice Kappa de 0,99. A matriz de confusão mostrou que um segmento não foi classificado de acordo com as classes de referência, configurando um erro de omissão. As classes que geraram maior confusão foram as classes mineração e de solo exposto. A classe mineração foi confundida com as de formação campestre e de afloramento. A classe de solo exposto foi confundida com a de formação campestre e/ou não foi classificada. A confusão entre solo exposto e formação campestre é explicada pela proximidade espacial e espectral. Ainda assim, ambas as classes tiveram um índice Kappa satisfatório, 0,98 e 0,96, respectivamente.

Para o ano de 2018, os valores da classificação foram de 92\% para a precisão geral e de 0,90 para o índice Kappa, demostrando um acordo quase perfeito entre a classificação realizada e a máscara de teste (LANDIS E KOCH, 1977). Foram identificadas pela matriz de confusão, que oito objetos não

$\begin{array}{lllll}\text { Caminhos de Geografia } \quad \text { Uberlândia-MG } & \text { v. 21, n. } 76 \quad \text { Ago/2020 } & \text { p. 57-71 } & \text { Página } 65\end{array}$


foram classificados de acordo com as classes de referência. Também para a imagem de 2018, as classes solo exposto e mineração foram as que apresentaram maior confusão. A classe de mineração foi confundida principalmente com a de afloramento rochoso e a classe de solo exposto foi confundida sobretudo com a classe de formação campestre. Apesar dos erros de comissão, advindas dessas classes, ambas apresentaram bons resultados no índice Kappa.

O mapeamento do uso e de cobertura da terra da bacia hidrográfica do rio Gualaxo do Norte dos anos de 2008, 2015 e 2018 estão apresentados na Figura 6.

Figura 6 - Mapeamento do uso e cobertura do solo da bacia hidrográfica do rio Gualaxo do Norte - MG nos anos de 2008, 2015 e 2018.

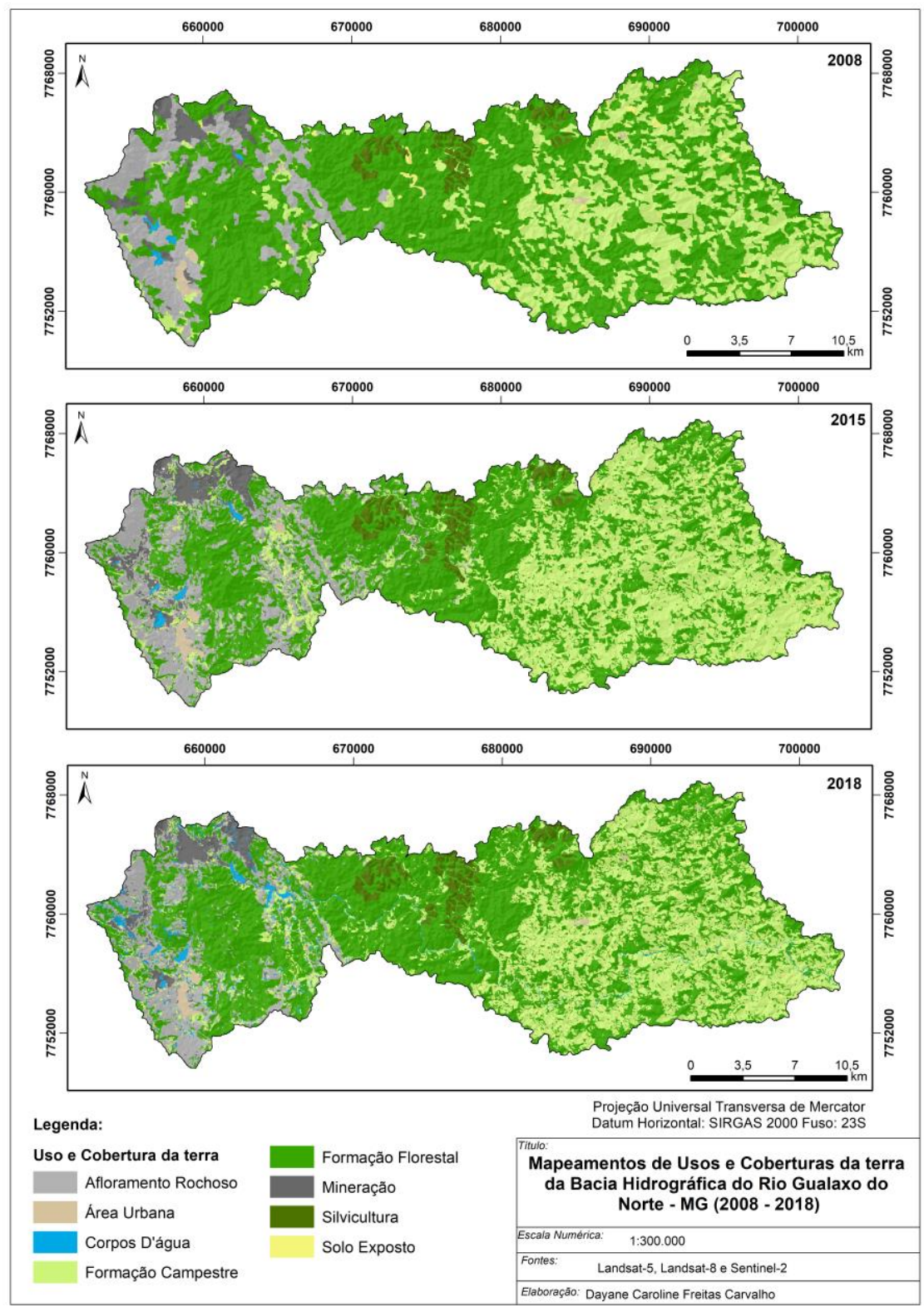

$\mathrm{Na}$ análise da dinâmica do uso e de cobertura da terra da área de estudo (Figura 6 e Tabela 7) no intervalo de 10 anos (2008 a 2018), foram observadas duas classes dominantes: a formação florestal e a formação campestre. A formação florestal é composta majoritariamente por matas secundárias em estágio inicial e médio de regeneração e concentra-se no médio curso da bacia. A abrangência da classe em 10 anos passou de 59,28\% em 2008 para 51,98\% em 2018. O desmatamento observado na bacia do rio Gualaxo do Norte evidencia um problema antigo enfrentado na bacia do rio Doce. Segundo Hora (2012), o desmatamento foi a base do estabelecimento de atividades agropecuárias que contribuíram, ao longo do tempo, para a degradação da bacia. A supressão da

$\begin{array}{lllll}\text { Caminhos de Geografia } \quad \text { Uberlândia-MG } & \text { v. 21, n. } 76 \quad \text { Ago/2020 } & \text { p. 57-71 } & \text { Página } 66\end{array}$ 
vegetação não foi, a princípio, especificamente motivada pela agropecuária, e sim para atender a demanda madeireira emergente, durante a primeira metade do século XX. Com o declínio da extração madeireira, por volta de 1950, a floresta tropical característica foi praticamente substituída por extensas áreas de pastagens, que permanecem até a atualidade.

Nesse sentido, destaca-se que as florestas exercem papel fundamental para o equilíbrio e adequado funcionamento dos ecossistemas e para a manutenção da diversidade biológica (BACELLAR, 2005). A supressão das mesmas promove alterações no meio físico e no clima e no sistema hidrológico da bacia (LINHARES; SOARES; BATISTA, 2005; BACELLAR, 2005). Sendo assim, a manutenção das formações florestais remanescentes, assim como a implementação de programas que levem à recomposição e restauração florestal na bacia são de fundamental importância para manutenção do equilíbrio ecossistêmico nessa região.

Tabela 7 - Abrangência espacial e percentual do mapeamento de uso e cobertura da terra da bacia hidrográfica do rio Gualaxo do Norte - MG, 2008 a 2018.

\begin{tabular}{|c|c|c|c|c|c|c|}
\hline Classe & $\begin{array}{c}2008 \\
\text { Área }\left(\mathrm{km}^{2}\right)\end{array}$ & $\begin{array}{l}2008 \\
\text { Área (\%) }\end{array}$ & $\begin{array}{c}2015 \\
\text { Área }\left(\mathrm{km}^{2}\right)\end{array}$ & $\begin{array}{l}2015 \\
\text { Área (\%) }\end{array}$ & $\begin{array}{c}2018 \\
\text { Área }\left(\mathrm{km}^{2}\right)\end{array}$ & $\begin{array}{c}2018 \\
\text { Área (\%) }\end{array}$ \\
\hline $\begin{array}{l}\text { Afloramento } \\
\text { Rochoso }\end{array}$ & 57,18 & 10,18 & 56,73 & 10,11 & 52,41 & 9,33 \\
\hline Área Urbana & 3,16 & 0,56 & 3,85 & 0,68 & 3,03 & 0,54 \\
\hline Corpos D’água & 1,31 & 0,23 & 1,79 & 0,32 & 9,92 & 1,77 \\
\hline $\begin{array}{l}\text { Formação } \\
\text { Campestre }\end{array}$ & 138,59 & 24,68 & 185,73 & 33,07 & 161,23 & 28,70 \\
\hline $\begin{array}{l}\text { Formação } \\
\text { Florestal }\end{array}$ & 332,96 & 59,28 & 278,08 & 45,50 & 291,97 & 51,98 \\
\hline Mineração & 12,10 & 2,16 & 16,75 & 2,98 & 14,52 & 2,59 \\
\hline Silvicultura & 11,81 & 2,10 & 13,91 & 2,48 & 14,40 & 2,56 \\
\hline Solo Exposto & 4,57 & 0,81 & 4,84 & 0,86 & 14,20 & 2,53 \\
\hline Total & 561,68 & 100 & 561,68 & 100 & 561,68 & 100 \\
\hline
\end{tabular}

As áreas de afloramento rochoso, de mineração e de barragens de rejeito concentram-se no alto curso da bacia. O decréscimo da classe afloramento rochoso é explicada pelo avanço da mineração nas cabeceiras da bacia. Silva (2007) considera a mineração como uma das atividades econômicas mais impactantes sobre o ambiente. No caso da bacia do rio Gualaxo do Norte, a ausência de um planejamento sustentável por parte das mineradoras, do poder público e da sociedade civil, resultou no rompimento da barragem de Fundão, comprometendo toda a bacia hidrográfica do rio Doce, gerando inúmeros impactos negativos significativos, tais como a degradação visual da paisagem, a redução da qualidade da água, do solo, do ar, além de impactos na flora e na fauna da bacia.

É irrefutável o papel da atividade minerária na bacia do Gualaxo do Norte e em todo estado de Minas Gerais. Segundo o Instituto Brasileiro de Mineração (2016), aproximadamente 4,5\% do PIB mineiro e $80 \%$ do PIB do município de Mariana, que compreende grande parte da bacia do rio Gualaxo do Norte, são derivados da indústria extrativa mineral, sem considerar petróleo e gás. Entretanto, a mineração apenas pode ser considerada sustentável se minimizar os seus impactos ambientais e mantiver certos níveis de proteção ecológica e de padrões de qualidade ambientais, além de garantir o bem-estar socioeconômico no presente (ENRÍQUEZ; FERNANDES; ALAMINO, 2011).

Alguns corpos d'água estão dentro de áreas de mineração. Esse tipo de evento é comum, pois várias minas formam uma espécie de lago nas cavas, onde a água fica retida. Comparando as áreas de 2008 e 2015, houve um pequeno acréscimo devido ao aumento da atividade minerária local, aumentando também a quantidade de água nas cavas. No ano de 2018, existe um grande aumento na área da mesma classe. Este aumento é explicado pela base cartográfica utilizada para o mapeamento. No mapeamento de 2018, foi utilizada uma imagem Sentinel-2 com uma resolução espacial de 10 metros. Assim, a calha do rio Gualaxo do Norte foi identificada, diferente dos outros anos, onde foram utilizadas imagens de 30 metros (Landsat 5 e 8) ou seja, com menor resolução espacial, sendo nestas identificadas apenas as classes referentes à borda do canal.

A silvicultura, no intervalo temporal estudado, sofreu um aumento gradual, saindo de $2,10 \%$, em 2008, para 2,56\%, em 2018, e representa um importante produto da economia regional. Em

$\begin{array}{lllll}\text { Caminhos de Geografia } \quad \text { Uberlândia-MG } & \text { v. 21, n. } 76 \quad \text { Ago/2020 } & \text { p. 57-71 } & \text { Página } 67\end{array}$


contrapartida, as áreas de solo exposto, no intervalo estudado, sofreram aumento de mais de $300 \%$. A classe, que representava $4,57 \mathrm{~km}^{2}$ em 2008, chegou a $14,2 \mathrm{~km}^{2}$, em 2018 . Tais áreas são mais suscetíveis a pressões antrópicas, necessitando de monitoramento e da adoção de práticas adequadas de manejo e conservação de solos. Assim, é possível evitar o carreamento de sedimentos para os cursos d'água e a formação de sulcos erosivos.

As áreas de solo exposto estão espacialmente localizadas nas proximidades das regiões de formação campestre, que são áreas com presença de pastagem, que aumentam a pressão sobre o solo. A formação campestre ocupa grande parte do baixo curso da bacia, e é uma classe importante no que se refere aos processos erosivos. Ela compreende as vegetações arbustivas e herbáceas, incluindo as pastagens (Figura 7).

As áreas de pastagem na região são constituídas, na sua maioria, por pastagens degradadas com ausência de práticas de manejo adequadas e apresentam baixa capacidade de suporte. São regiões que sofrem com o pisoteio do gado e, quando estão posicionadas em áreas de relevo mais movimentado, atuam como aceleradores dos processos erosivos.

Figura 7 - Pastagem no baixo curso da bacia hidrográfica do rio Gualaxo do Norte - MG.

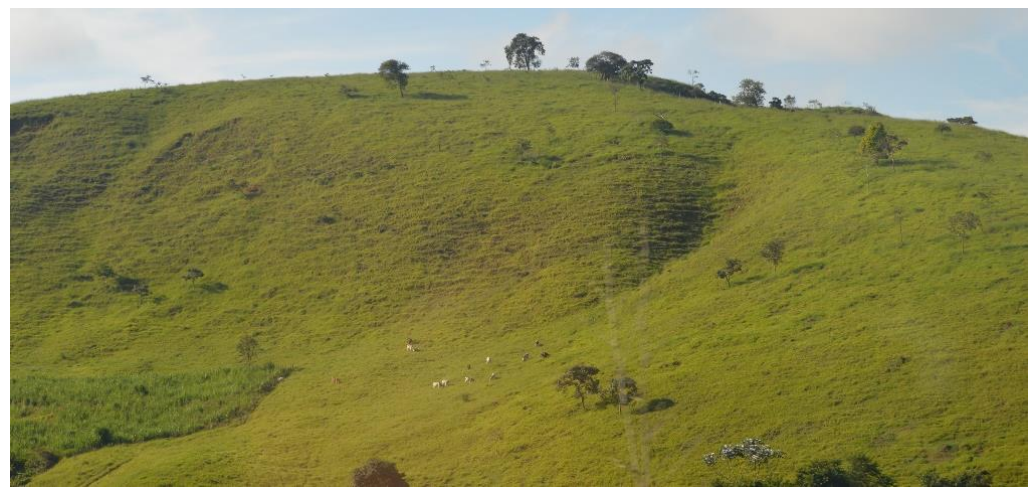

A classe de área urbana sofreu um pequeno aumento do ano de 2008 para o de 2015. Em 2018, ocorreu um decréscimo de aproximadamente $20 \%$ da área da classe. A bacia hidrográfica do rio Gualaxo do Norte não é historicamente marcada pela presença de grandes áreas urbanas, mas por pequenos distritos influenciados pela mineração, pela silvicultura e/ou pela pecuária. $O$ decréscimo dessas áreas é explicado pelo rompimento da barragem de rejeitos minerais de Fundão, que devastou algumas áreas urbanas como Bento Rodrigues, subdistrito do distrito de Santa Rita Durão, município de Mariana. Situado a pouco mais de $5 \mathrm{~km}$ das barragens e a $35 \mathrm{~km}$ da sede de Mariana, Bento Rodrigues foi parcialmente soterrado pela avalanche de água, lama e detritos produzida com o rompimento da barragem. No subdistrito, viviam cerca de 600 habitantes e segundo estimativas do IBAMA, em torno de 207 dos 251 imóveis foram destruídos naquela localidade (ANA, 2016).

\section{CONSIDERAÇÕES FINAIS}

A aplicação da OBIA em imagens de satélite com resolução espacial média permitiu obter resultados satisfatórios. Entretanto, é importante destacar que algumas classes apresentaram muita confusão na classificação e forçaram a vetorização manual das feições.

A bacia hidrográfica do rio Gualaxo do Norte - MG apresenta padrões particulares de organização de uso e cobertura da terra. A bacia, mesmo tendo passado pelo episódio catastrófico do rompimento da barragem de Fundão, ainda conserva a mesma tendência anterior de uso, ora demarcada pelas características ambientais, como nos casos das cabeceiras de drenagem, com a presença dos afloramentos rochosos onde ocorre a atividade minerária, ora demarcada por características históricas de ocupação, como as pastagens localizadas no baixo curso.

O diagnóstico apresentado nesse trabalho pode ajudar na elaboração de planos, de projetos e de programas para o direcionamento de ações na bacia. Serve como subsídio nas tomadas de decisão 
para discussões acerca de medidas de intervenção, de recuperação de áreas degradadas, de conservação, e na identificação de áreas prioritárias para implementação de ações na bacia.

\section{AGRADECIMENTOS}

À CAPES pela bolsa de Mestrado da primeira autora, ao Programa de Pós-graduação em Análise e Modelagem de Sistemas Ambientais e ao Laboratório de Solos e Meio Ambiente do IGC/UFMG pela disponibilização de estrutura física e financeira para realização deste estudo.

\section{REFERÊNCIAS}

ANA - Agencia Nacional das Águas. Encarte especial sobre a bacia do rio Doce rompimento da barragem em Mariana MG. Conjuntura dos recursos hídricos no Brasil. Brasília - DF.2016. 50p.

BAATZ, M.; SCHAPE, A. Multiresolution Segmentation: An Optimization Approach for High Quality Multi-Scale Image Segmentation. In: Strobl, J., Blaschke, T. and Griesbner, G., Eds., Angewandte Geographische Informations-Verarbeitung, XII, Wichmann Verlag, Karlsruhe, Germany, p. 12-23, 2000.

BACELLAR, Luiz de A. P. O papel das florestas no regime hidrológico de bacias hidrográficas. Geo.Br. Ouro Preto, n.1, pp. 1-39. 2005.

BRASIL. Lei ํo 12.651, de 25 de maio de 2012. Dispõe sobre a proteção da vegetação nativa; altera as Leis nos 6.938, de 31 de agosto de 1981, 9.393, de 19 de dezembro de 1996, e 11.428, de 22 de dezembro de 2006; revoga as Leis nos 4.771, de 15 de setembro de 1965, e 7.754, de 14 de abril de 1989, e a Medida Provisória no 2.166-67, de 24 de agosto de 2001; e dá outras providências. Disponível em: Acesso em: 25/01/2019.

CAI, S.; LIU, D. A comparison of object-based and contextual pixel-based classifications using high and medium spatial resolution images. Remote Sensing. Lett. 2013, 4, p. 998-1007. Disponível em: https://www.tandfonline.com/doi/abs /10.1080/2150704X.2013.828180?journalCode=trsl20 Acesso em: 20 jan 2018. https://doi.org/10.1080/2150704X.2013.828180

CARMO. V. A Contribuição da etnopedologia para o planejamento das terras: estudo de caso de uma comunidade de agricultores do entorno do Parque Nacional do Caparaó. 2009. 197p. Tese (Doutorado em Geografia) - Instituto de Geociências, Universidade Federal de Minas Gerais, Belo Horizonte,

2009.

COELHO. A. L. N. Alterações Hidrogeomorfológicas no Médio-baixo Rio Doce / ES. 2007. 227p. Tese (Doutorado em Geografia) - Universidade Federal Fluminense, Universidade Federal Fluminense, Niterói, 2007.

CONGALTON, R. G.; GREEN, K. Assessing the accuracy of remotely sensed data: principles and practices. New York: Lewis Publishers, 1999. 136 p. https://doi.org/10.1201/9781420048568

CONSÓRCIO ECOPLAN-LUME. PARH - Plano de ação de recursos hídricos da unidade de planejamento e gestão DO1. 2010. 124 p. Disponível em: http://www.cbhdoce.org.br/wpcontent/uploads/2014/10/PARH_Piranga.pdf Acesso em: 20 de janeiro de 2019.

DEFINIENS. Ecognition developer 8: reference book. Definiens AG, Trappentreustr. 1, München, Germany 276 p, 2009.

DORREN, L.K.A.; MAIER, B.; SEIJMONSBERGEN, A.C. Improved Landsat-based forest mapping in steep mountainous terrain using object-based classification. Forest Ecology Management. 2003, 183, 31-46. https://doi.org/10.1016/S0378-1127(03)00113-0

EMBRAPA - Empresa Brasileira de Pesquisa Agropecuária. Serviço nacional de levantamento e conservação de solos. Rio de Janeiro: Serviço Nacional de Levantamento e Conservação de Solos/Embrapa-SNLCS. Micelânea, 1, 1979. 83 p.

ENRÍQUEZ, M. A. R.; FERNANDES, F. R. C.; ALAMINO, R. C. J. A mineração das grandes minas e as dimensões da sustentabilidade. IN: FERNANDES, F. R. C.; ENRÍQUEZ, M. A. R; ALAMINO, R. C. J. (orgs). Recursos naturais e sustentabilidade natural. Grande Minas. v.1. Rio de Janeiro. 2011. 
ESA - European Space Agency. Sentinel-2. Disponível em: https://sentinel.esa.int/web/sentinel/missions/sentinel-2. Acesso em: 20 jun 2018.

ESRI - Environmental Systems Research Institute. ArcGIS Release 10.3. Redlands, CA, 2014.

FLANDERS, D.; HALL-BEYER, M.; PEREVERZOFF, J. Preliminary evaluation of eCognition objectbased software for cut block delineation and feature extraction. Canadian Journal Remote Sensing. 2003 , 29 , 441-452.

https://doi.org/10.5589/m03-006

GILBERTSON, J.K.; KEMP, J.; VAN NIEKERK, A. Effect of pan-sharpening multi-temporal Landsat 8 imagery for crop type differentiation using different classification techniques. Computers Electronics in Agriculture. 2017, 134, 151-159. https://doi.org/10.1016/j.compag.2016.12.006

GOOGLE EARTH PRO. Acervo de imagens orbitais 2017 Disponível em: $<$ https://www.google.com/earth/>. Acesso em: 20 Jan 2018.

HORA, A. M. Da exploração econômica da bacia hidrográfica do rio doce ao processo de degradação de seus recursos naturais. In: GUEDES, G. R.; O. R. (orgs.). Território, mobilidade populacional e ambiente. 2012. Governador Valadares: Ed. Univale. p. 201-234.

HUSSAIN, M.; CHEN, D.; CHENG, A.; WEI, H.; STANLEY, D. Change detection from remotely sensed images: From pixel-based to object-based approaches. ISPRS Journal of Photogrammetry Remote Sensing. 2013, 80, 91-106. https://doi.org/10.1016/j.isprsjprs.2013.03.006

IBGE - Instituto Brasileiro ee Geografia e Estatística. Bases Cartográficas. Rio de Janeiro: IBGE, 2006. Disponível em: https://mapas.ibge.gov.br/bases-e-referenciais/bases-cartograficas/malhasdigitais.html Acesso em 03/03/2018.

Minas Gerais - Mariana. Instituto Brasileiro de Geografia Estatística: Brasília, 2010. Disponível em: Acesso em 28 de janeiro de 2019.

IBRAM - Instituto Brasileiro de Mineração. Panorama da Mineração em Minas Gerais. Instituto Brasileiro de Mineração, Sindicato Nacional da Indústria da Extração do Ferro de Metais - Brasília: IBRAM, 2015. $280 \mathrm{f}$.

IGAM - Instituto Mineiro de Gestão das Águas. Unidades de Planejamento e Gestão de Recursos Hídricos Minas 2010.2 Gerais. 20 Disponível http://www.igam.mg.gov.br/images/stories/mapoteca/upgrhs-minas-gerais.pdf Acesso em: 03 de mar de 2018.

INPE - Instituto Nacional de Pesquisas Espaciais. Departamento de Geração de Imagens. Disponível em: http://www.dgi.inpe.br/. Acesso em: 20 jun 2018.

JAXA/METI. Alos Palsar Wbi, 2010. Disponível em: <https://www.asf.alaska.edu>. Acesso em 20 jun 2018.

LACAZ. F. A. C. PORTO, M. F. S. PINHEIRO. T. M. M. Tragédias brasileiras contemporâneas: o caso do rompimento da barragem de rejeito de Fundão/Samarco. Revista Brasileira de Saude Ocupacional. [online]. 2017; 42:e9. Disponível em: https://www.scielo.br/scielo.php?script=sci_issuetoc\&pid=0303765720170001\&lng=pt\&nrm=iso Acesso em: 20 de jan de 2019. https://doi.org/10.1590/2317$\underline{6369000016016}$

LANDIS, J.R. e KOCH, G.G. The measurement of observer agreement for categorical data. Biometrics, v.33, n.1, p. 159-174, 1977. https://doi.org/10.2307/2529310

LI, M.; ZANG, S.Y.; ZHANG, B.; LI, S.S.; WU, C.S. A review of remote sensing image classification techniques: The role of spatio-contextual information. European Journal Remote Sensing. 2014, 47, p. 389-411. https://doi.org/10.5721/EuJRS20144723

LINHARES, C. A.; SOARES, J. V.; BATISTA, G. T. Influência do Desmatamento na dinâmica da resposta hidrológica da bacia do Ji-Paraná. Anais... XII Simpósio Brasileiro de Sensoriamento Remoto, 2005, Goiânia (GO). Anais do XII Simpósio Brasileiro de Sensoriamento Remoto, 2005, INPE, p.3097-3105.

MOSKAL, L.M.; STYERS, D.M.; HALABISKY, M. Monitoring urban tree cover using object-based image analysis and public domain remotely sensed data. Remote Sensing. Basel, Switzerland. 2011, 3, 2243-2262. https://doi.org/10.3390/rs3102243 
PEÑA, J.M.; GUTIÉRREZ, P.A.; HERVÁS-MARTíNEZ, C.; SIX, J.; PLANT, R.E.; LÓPEZGRANADOS, F. Object-based image classification of summer crops with machine learning methods. Remote Sensing. Basel, Switzerland. 2014, 6, p. 5019-5041. https://doi.org/10.3390/rs6065019

PHIRI, D. MORGENROTH, J. Developments in Landsat land cover classification methods: a review. Remote Sensing. Basel, Switzerland. 2017, 9, 967. https://doi.org/10.3390/rs9090967

PIAZZA, G. A.; VIBRANS, A. C. classificação orientada a objetos para mapear florestas secundárias em Santa Catarina, sul do Brasil. Revista Brasileira de Cartografia, v. 66, n. 5, 3 nov. 2014.

QGIS Development Team. QGIS User Guide, Release 2.18: QGIS Project, 2016. Disponível em: https://www.qgis.org/pt_BR/site/forusers/download.html Acesso em: 20 de jun de 2018

ROSA, R. Introdução ao sensoriamento remoto. Uberlândia. Ed. UFU, 2007. 248p. https://doi.org/10.14393/EDUFU-85-7078-124-6

SAADI, A.; CAMPOS, J. C. F. Geomorfologia do caminho da lama: contexto e consequências da ruptura da Barragem do Fundão (novembro 2015-Mariana-MG). Revista Arquivos do Museu de História Natural e Jardim Botânico da UFMG. Belo Horizonte, v. 24, p. 63-103, 2015.

SAMAL, D. R.; GEDAM, S.S. Monitoring land use changes associated with urbanization: An object based image analysis approach. European Journal Remote Sensing. 2015, 48, 85-99. https://doi.org/10.5721/EuJRS20154806

SANTOS, J, P. et al. Susceptibilidade à erosão no Rio Gualaxo do Norte - MG. Caminhos de Geografia. Uberlândia, MG. v.18 n.63. set. 2017. https://doi.org/10.14393/RCG186313

SILVA, João Paulo Souza. Impactos ambientais causados por mineração. Revista Espaço da Sophia. [online]. 2007, vol.1, n.8. ISSN 1981-318X.

TRIMBLE. Software eCognition Developer Trial 8.0. 1995-2011. Disponível em: < http://www.ecognition.com/>.

USGS - United States Geological Survey Missões Landsat. Disponível em: https://www.usgs.gov/landresources/nli/landsat/landsat-8?q science_support_page_related_con. Acesso em: Acesso em: 20 jun 2018.

VIANA, J. H. M. COSTA, A. M. Desastre de Mariana e dos solos da Bacia do Rio Doce. Boletim informativo Sociedade Brasileira de Ciência do Solo. vol.42, n. 1 (jan./abr. 2016). Viçosa: SBCS. 2016.

Recebido em: 05/04/2019

Aceito para publicação em: 12/06/2020 